OPEN ACCESS

Edited by:

Enzo Lalli,

UMR7275 Institut de pharmacologie moléculaire et cellulaire (IPMC), France

Reviewed by: Alfredo Campenni', University of Messina, Italy Christian Albert Koch, Fox Chase Cancer Center, United States

${ }^{*}$ Correspondence:

Christina Lenschow Lenschow_C@ukw.de

Specialty section: This article was submitted to Cancer Endocrinology, a section of the journal Frontiers in Endocrinology

Received: 17 December 2020 Accepted: 04 February 2021 Published: 23 March 2021

Citation:

Lenschow C, Fuss CT, Kircher S, Buck A, Kickuth R, Reibetanz J,

Wiegering $A$, Stenzinger $A$, Hübschmann D, Germer CT, Fassnacht M, Fröhling S, Schlegel N and Kroiss M (2021) Case Report: Abdominal Lymph Node Metastases of Parathyroid Carcinoma: Diagnostic

Workup, Molecular Diagnosis, and Clinical Management.

Front. Endocrinol. 12:643328. doi: 10.3389/fendo.2021.643328

\section{Case Report: Abdominal Lymph Node Metastases of Parathyroid Carcinoma: Diagnostic Workup, Molecular Diagnosis, and Clinical Management}

\author{
Christina Lenschow ${ }^{1 *}$, Carmina Teresa Fuss ${ }^{2}$, Stefan Kircher ${ }^{3}$, Andreas Buck ${ }^{4}$, \\ Ralph Kickuth ${ }^{5}$, Joachim Reibetanz ${ }^{1}$, Armin Wiegering ${ }^{1}$, Albrecht Stenzinger ${ }^{6,7}$, \\ Daniel Hübschmann ${ }^{8}$, Christoph Thomas Germer ${ }^{1}$, Martin Fassnacht ${ }^{2,9}$, Stefan Fröhling ${ }^{10}$, \\ Nicolas Schlegel ${ }^{1}$ and Matthias Kroiss ${ }^{2,9,11}$
}

\begin{abstract}
1 Department of General, Visceral, Transplant, Vascular and Pediatric Surgery, University Hospital Würzburg, University of Würzburg, Würzburg, Germany, ${ }^{2}$ Department of Internal Medicine I, Division of Endocrinology and Diabetes, University Hospital, University of Würzburg, Würzburg, Germany, ${ }^{3}$ Institute of Pathology, University of Würzburg, Würzburg, Germany, ${ }^{4}$ Department of Nuclear Medicine, University Hospital Würzburg, University of Würzburg, Würzburg, Germany, ${ }^{5}$ Department of Diagnostic and Interventional Radiology, University Hospital Würzburg, University of Würzburg, Würzburg, Germany, ${ }^{6}$ Institute of Pathology, University Hospital Heidelberg, Heidelberg, Germany, ${ }^{7}$ Germany and German Cancer Consortium (DKTK), Heidelberg Partner Site, Heidelberg, Germany, ${ }^{8}$ Computational Oncology, Molecular Diagnostics Program, NCT Heidelberg and Heidelberg University Hospital, Heidelberg, Germany, ${ }^{9}$ Comprehensive Cancer Center Mainfranken, University of Würzburg, Würzburg, Germany, ${ }^{10}$ National Center for Tumor Diseases (NCT Heidelberg), Division of Translational Medical Oncology German Cancer Research Center (DKFZ), University Hospital Heidelberg, Heidelberg, Germany, ${ }^{11}$ Department of Medicine IV, University Hospital Munich, Ludwig-Maximilians-Universität München, Munich, Germany
\end{abstract}

Parathyroid carcinoma $(\mathrm{PC})$ is an orphan malignancy accounting for only $~ 1 \%$ of all cases with primary hyperparathyroidism. The localization of recurrent PC is of critical importance and can be exceedingly difficult to diagnose and sometimes futile when common sites of recurrence in the neck and chest cannot be confirmed. Here, we present the diagnostic workup, molecular analysis and multimodal therapy of a 46-year old woman with the extraordinary manifestation of abdominal lymph node metastases 12 years after primary diagnosis of PC. The patient was referred to our endocrine tumor center in 2016 with the aim to localize the tumor causative of symptomatic biochemical recurrence. In view of the extensive previous workup we decided to perform [18F]FDG-PET-CT. A pathological lymph node in the liver hilus showed slightly increased FDG-uptake and hence was suspected as site of recurrence. Selective venous sampling confirmed increased parathyroid hormone concentration in liver veins. Abdominal lymph node metastasis was resected and histopathological examination confirmed PC. Within four months, the patient experienced biochemical recurrence and based on high tumor mutational burden detected in the surgical specimen by whole exome sequencing the patient received immunotherapy with pembrolizumab that led to a biochemical response. Subsequent to disease progression repeated abdominal lymph node resection was performed in 10/ $2018,01 / 2019$ and in $01 / 2020$. Up to now (12/2020) the patient is biochemically free of 
disease. In conclusion, a multimodal diagnostic approach and therapy in an interdisciplinary setting is needed for patients with rare endocrine tumors. Molecular analyses may inform additional treatment options including checkpoint inhibitors such as pembrolizumab.

Keywords: parathyroid carcinoma, abdominal lymph node metastases, molecular diagnostics, repeated surgery, [18F]FDG-PET-CT, immune check inhibitor, pembrolizumab

\section{INTRODUCTION}

Parathyroid carcinoma PC is an orphan malignancy occurring in approximately 1\%-5\% (United States, Europe, Japan) of all patients with primary hyperparathyroidism (pHPT) (1-5). The main preoperative challenge of $\mathrm{PC}$ is to raise the suspicion of malignant disease at diagnosis since clinical outcome and prognosis are largely dependent on the extent of primary surgery. Despite the combination of multiple diagnostic modalities, this rare tumor is often difficult to diagnose preoperatively (6-9). Moreover, diagnosis of malignancy is made in only $15 \%$ of the fast-frozen sections. So, in the vast majority of cases, only the final histology or a relapse provides the diagnosis (10).

The high rate of relapse is another considerable problem in PC patients. We have recently published a comprehensive clinical characterization of $83 \mathrm{PC}$ cases and have demonstrated that within a median interval of 48 months $38.6 \%$ of cases relapsed (7). However, in case of biochemical recurrence, the precise localization of cancerous tissue is mandatory to enable surgical treatment. The calcimimetic drug cinacalcet is approved to control serum calcium and may be used in case of unsuccessful localization and/or advanced, non-resectable disease. Systemic antitumoral therapy has remained anecdotal (11).

To date, only very few cases of PC with abdominal tumor localization (peripancreatic lymph nodes: $n=1$, liver $n=6$ ) have been described (12-14).

Here, we present the complex diagnostic workup and multimodal therapy in a 46-year old woman with the uncommon manifestation of abdominal metastases of PC.

\section{CASE DESCRIPTION}

A 46-year old woman was referred to our endocrine tumor center in 2016 with the aim to localize the tumor causative of biochemical recurrence.

Primary surgery due to pHPT had been performed in 2004. Intraoperatively, PC was suspected and en-bloc-resection (hemithyroidectomy, parathyroidectomy and central lymph node dissection of the left side) was performed. PC was confirmed histopathologically and resection margins were free of tumor (R0). The patient experienced a permanent recurrent laryngeal nerve palsy at the left side as complication of the surgical procedure. She underwent postoperative adjuvant external beam radiation of the thyroid region at a total dose of $50.4 \mathrm{~Gy}$. The patient was subsequently free of disease for 12 years.

In 2016, the patient experienced symptoms similar to those at initial diagnosis with thirst, fatigue and visual flashes. Serum calcium was elevated up to $3.4 \mathrm{mmol} / \mathrm{L}$ [reference range: 2.0 $2.7 \mathrm{mmol} / \mathrm{L}]$ and parathyroid hormone $(\mathrm{PTH})$ was increased to $203 \mathrm{pg} / \mathrm{mL}$ [10-65pg/ml]. Medication with cinacalcet was initiated. Within the three subsequent months, the patient underwent cervical ultrasonography, CT and MRI of the neck and chest, $\left[{ }^{99 \mathrm{~m}} \mathrm{Tc}\right.$ ] Sestamibi-SPECT/CT, as well as [11C] methionine-PET-CT. None of these imaging modalities localized tumor recurrence. Additionally, the patient underwent reexploration of the right neck and lymph node dissection of the right cervical lymph nodes. Histopathology was negative for PC and hypercalcemia persisted postoperatively.

Subsequently the patient presented herself at our institution. In view of the extensive previous workup we decided to perform [18F] FDG-PET-CT. Surprisingly, a pathological lymph node $(17 \times 24$ $\mathrm{mm}$ ) within the liver hilus showed slightly increased FDG-uptake (Figures 1A, B). Due to the unusual localization we questioned relationship with the recurrence of PC and performed selective PTH venous sampling that included the entire neck region, but also sampling from the liver veins in addition to V. cava inferior sampling (Figure 1C). Highest PTH was measured in the right V. hepatica with $758 \mathrm{pg} / \mathrm{ml}$ and a ratio of 1.3 compared to $\mathrm{V}$. cava inferior. After laparotomy and preparation of the liver hilus, the lymph node was resected with intact capsule without any signs of infiltration (R0) in January 2017. Inspection revealed no further lymph node manifestations (Figures 1D, E). Intraoperative PTH dropped from $841 \mathrm{ng} / \mathrm{L}$ to $387 \mathrm{ng} / \mathrm{L}$ The peri-and postoperative course was without any complications. The histological assessment of the resected tissue confirmed lymph node metastasis with blood vessel infiltration (V1) of PC (Figure 1F). Histological and immunohistochemical analyses were both consistent with parathyroid carcinoma (positivity for $\mathrm{PTH}$, loss of parafibromin). Furthermore, we stained the lymph node sample for PD-L1 (antibody 28-8). In the tumor cell there was no specific membrane-bound positivity (TC Score 0; Cologne -Score for NSCLS) (15). No tumor associated PD-L1 positivity in the background inflammatory infiltrate was observed. Moreover, we analyzed the DNAMismatch-Repair-Protein MLH1, MSH2, MSHG, and PMS2. The results showed no evidence of microsatellite instability. In summary, this was a lymph node metastases of a PD-L1-negative, microsatellite stable parathyroid carcinoma.

After resection, Calcium decreased steadily from $3.4 \mathrm{mmol} / \mathrm{L}$ to $2.3 \mathrm{mmol} / \mathrm{L}$. Cinacalcet was discontinued.

\section{SYSTEMIC TREATMENT}

Unfortunately, few days after discharge, PTH level and Calcium level increased slowly again requiring the use of cinacalcet. 

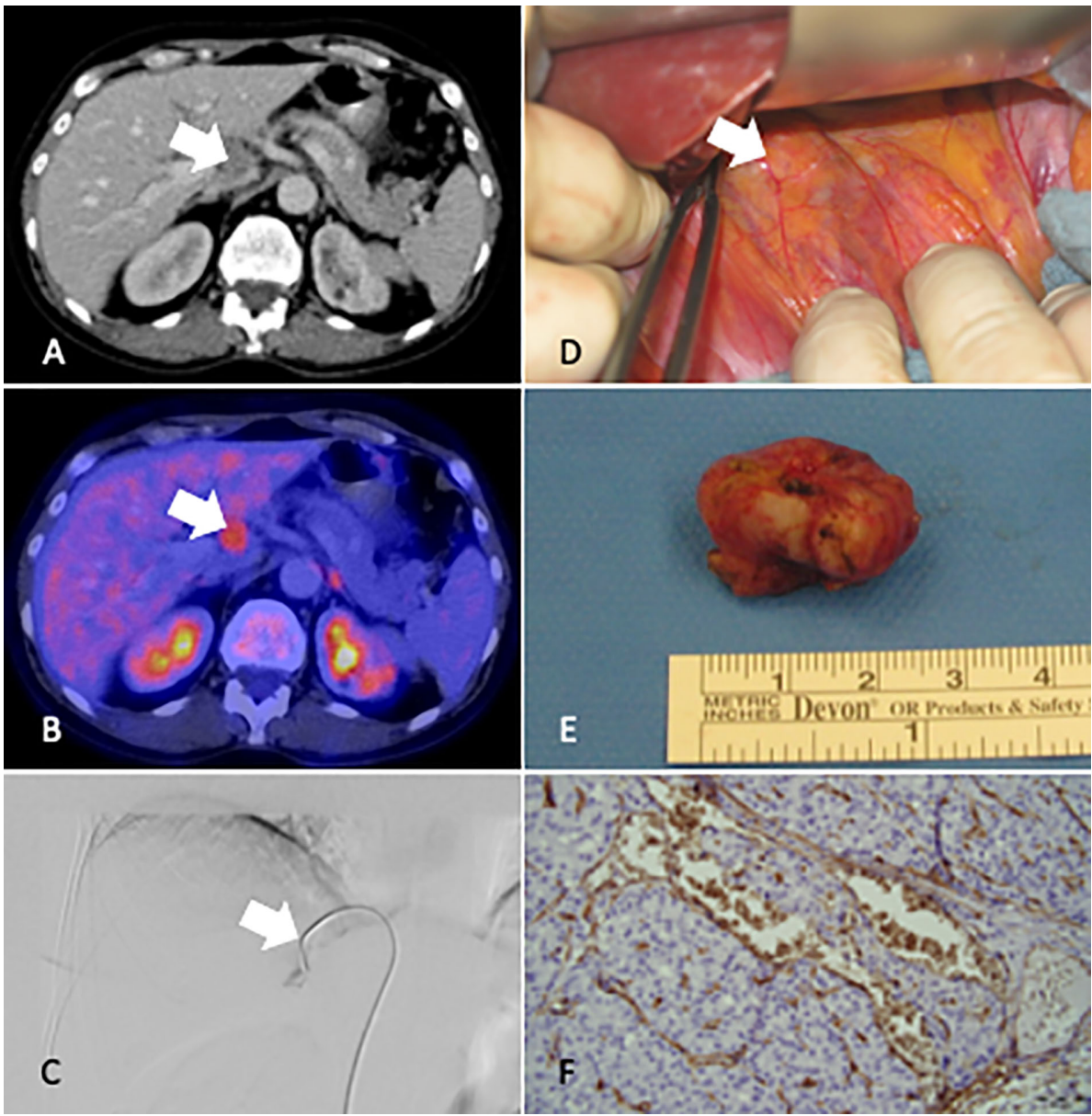

FIGURE 1 | CT Imaging (A), [18F]FDG-PET-CT (B), Venous sampling V. hepatica (C), intraoperative localization (D), Resected lymph node (E), histological result at time of diagnosis recurrence $\mathbf{( F )}$, White arrow marks the lymph node metastases in the hilus of the liver.

The medication was increased to a daily cumulative dose of $150 \mathrm{mg}$ in the following weeks. Four months after resection, PTH rose to $912 \mathrm{ng} / \mathrm{L}$ and additionally, denosumab was required to adequately control serum calcium levels $(2.6 \mathrm{mmol} / \mathrm{l})$.

Abdominal ultrasonography indicated recurrent lymph node metastases in the hepatic hilus (Figure 2A) which was confirmed by [18F]FDG-PET-CT.

The patient was included in NCT/DKTK MASTER (Molecularly Aided Stratification for Tumor Eradication Research), a multicenter, prospective observational study that is based on a common workflow for diagnostics, therapeutic decision making, and structured follow-up in patients with rare tumors failing standard treatment. The formalin-fixed paraffin embedded lymph node tissue obtained at surgery was utilized for whole exome sequencing and 412 single nucleotide variants (SNV) and 2 insertions/deletions (indels) were identified.

Because of the rapid recurrence in the same location, the fact that PTH levels had not returned to normal levels after resection of the previous metastases and in view of controlled serum calcium ( $2.6 \mathrm{mmol} / \mathrm{L}$ ) only with $150 \mathrm{mg}$ cinacalcet, we decided to initiate therapy with pembrolizumab on a compassionate use basis at 200 mg every three weeks. Pembrolizumab treatment was started in 09/2017 at a PTH serum concentration of 1,906 ng/L (Figure 3F). After four cycles of therapy, PTH dropped significantly (11/2017) to $613 \mathrm{ng} / \mathrm{L}$ and [18F]FDG-PET-CT showed stable disease four months later. Pembrolizumab was continued for six infusions every three weeks until [18F]FDG-PET-CT detected a lymph node bulk adjacent to the gallbladder (Figure 2B) in 07/2018. After evaluation of resectability, re-laparotomy was performed (10/ 2018) and the lymph node conglomerate of $3.4 \times 2.0 \times 3.2 \mathrm{~cm}$ in lymph node station 12 was resected. There were no clinical signs of extra nodal tumor infiltration (Figures 2C, D). Intraoperative PTH dropped from $2864 \mathrm{ng} / \mathrm{L}$ to $64.3 \mathrm{ng} / \mathrm{L}$. Histology confirmed a conglomerate of lymph node metastases of the PC up to $3.8 \mathrm{~cm}$ in size exhibiting the same features as the initial specimen. The postoperative course of the patient was unremarkable. In the following months, the patient's serum calcium and PTH levels increased slowly. The patient was normocalcaemic on intermittent medication with denosumab and had stable PTH levels between 197 und $332 \mathrm{pg} / \mathrm{L}$ until 06/2019. At this time a progress of 




FIGURE 2 | Abdominal ultrasonography (A) [18F]FDG-PET-CT (B), photography surgery 10/2018, Lymph node metastases in situ (C) (white arrows mark the tumor), and the tumor localization in an anatomical drawing (D). CD, cystic duct; CBD, common bile duct; CHD, common hepatic duct.

increased serum calcium levels $(2.8 \mathrm{mmol} / \mathrm{L})$ and PTH $(418 \mathrm{ng} / \mathrm{L})$ was detected. A [18F]FDG-PET-CT was performed and showed a new lymph node recurrence near the diaphragm (Figures 3A-D). This lymph node was resected as well as a lymph node dorsal of the V. cava inferior in $01 / 2020$ (Figure 3E). PTH dropped intraoperatively to $15.8 \mathrm{ng} / \mathrm{L}$ (preoperative: $1,831 \mathrm{ng} / \mathrm{L}$ ). Due to postoperative hypocalcemia, the patient received decostriol $(1,25$ dihydroxycholecalciferol) at a dose of $0.5 \mu \mathrm{g}$ twice a day and up to $3 \mathrm{~g}$ calcium P.O. per day which could be discontinued during the following 8 weeks. Until 10/2020 the patient was biochemically free of recurrence (serum calcium $2.4 \mathrm{mmol} / \mathrm{L}, \mathrm{PTH} 42.7 \mathrm{pg} / \mathrm{ml}$ ) without any medication. The whole course of PTH is shown in Figure 3F. Interdisciplinary discussion recommended watchful waiting and restaging in case of biochemical progression.

\section{DISCUSSION}

The diagnostic workup and site directed therapy in case of recurrent PC remains challenging. Distant metastases and especially abdominal localization of PC, as presented in this case report, are highly uncommon (7). The review of the literature revealed case reports of distant metastases, specifically abdominal and brain metastases (12-14) (Table 1). Interdisciplinary management is crucial to enable focused surgical treatment and adequate medical treatment (16). In this case report, we presented an example for an interdisciplinary workflow in a patient with abdominal recurrent PC 12 years after primary diagnosis.

\section{[18F]FDG-PET-CT AND SELECTIVE VENOUS SAMPLING IMPROVES LOCALIZATION DIAGNOSTICS}

After biochemical recurrence in our patient, tumor localization was not possible by various imaging techniques. However, [18F] FDG-PET-CT was able to visualize the unusual localization of abdominal recurrence. In several a case series, [18F]FDG-PETCT has been shown to effectively localize PC manifestation sites at initial diagnosis, follow-up or recurrence (17). In the current literature, $[18 \mathrm{~F}]$ choline-PET-CT was compared in small series with [18F]FDG-PET-CT. In 2 cases [18F]FDG-PET-CT detected tumor manifestations in the liver and bone lesions in the pelvis, which was missed by [18F]FDG-PET-CT. The authors of that study discussed that the differences in choline and FDG uptake could be the differences in tumor proliferation and differentiation (18). Another case report demonstrated that [18F]FDG-PET-CT and [18F]choline-PET-CT are feasible, in (recurrent) PC (19). In summary, as long as there is no standardized diagnostic work-up especially in recurrent PC, whole body functional imaging should be considered for 

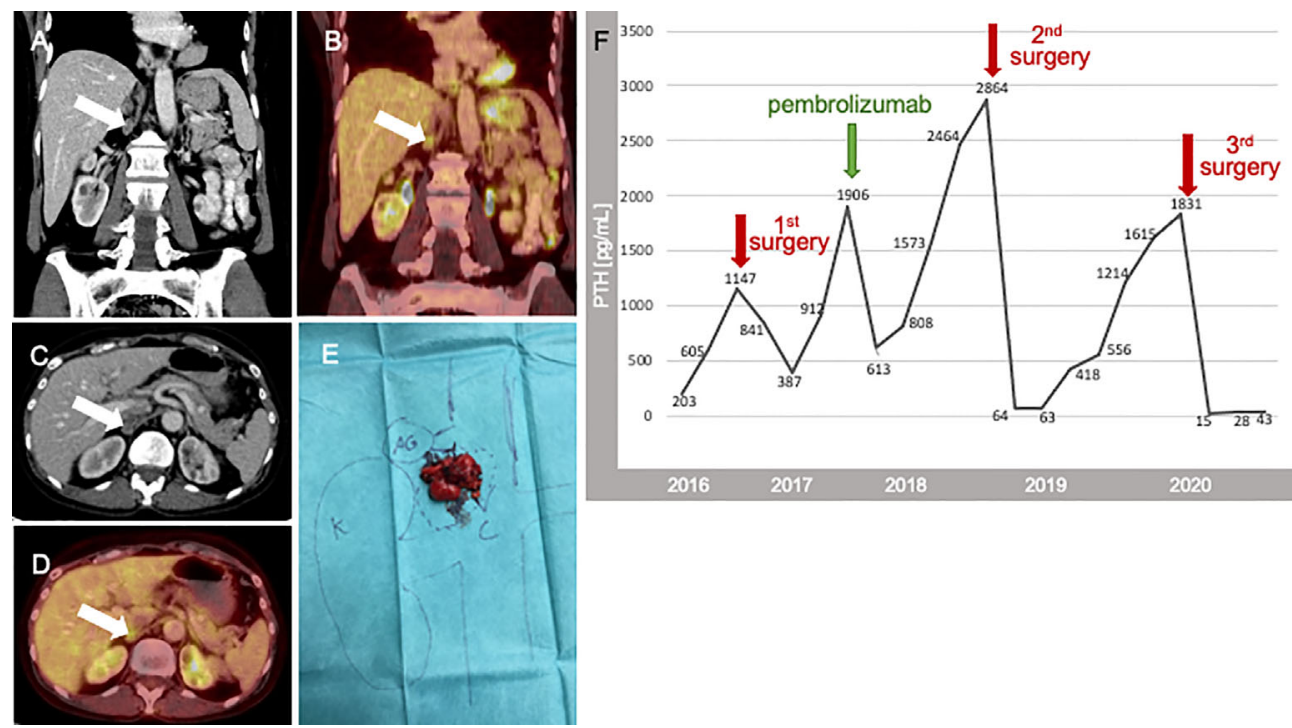

FIGURE 3 | CT Imaging (A, C), 12/2019), [18F]FDG-PET-CT (B, D), (white arrows mark the tumor), photography surgery 01/2020 E; K, Kidney; VC, Vena cava; AG, adrenal gland; PTH course is shown as time scale from the beginning of treatment in our hospital 2016 up to 2020 . In this period, we performed three abdominal surgeries (red arrow) and the beginning of therapy within Pembrolizumab (green arrow) (F).

TABLE 1 | Unusual localizations of distant PC recurrence in the literature.

\begin{tabular}{|c|c|c|c|c|c|c|c|}
\hline First Authors & $\begin{array}{c}\text { Publication } \\
\text { Year }\end{array}$ & $\begin{array}{l}\text { Patients } \\
\qquad(n=)\end{array}$ & Sites of Distant Metastases (DM) & $\begin{array}{c}\text { Finding Modality } \\
\text { of DM }\end{array}$ & Treatment of DM & Outcome & $\begin{array}{c}\text { Follow up } \\
\text { (years) }\end{array}$ \\
\hline Manente et al. & 1987 & 1 & Lymph node Pancreas region & Not Reported & Resection & Death & 1 \\
\hline \multirow[t]{3}{*}{ Qiu Zhong-ling et al. } & 2013 & 5 & Liver & $\begin{array}{l}\text { CT } n=5 \\
\text { Biopsy } n=3\end{array}$ & $\begin{array}{l}\text { RFA /EB } n=1 \\
P R n=3\end{array}$ & $\begin{array}{l}\text { Alive } n=3 \\
\text { Death } \\
n=2\end{array}$ & $0.5-9$ \\
\hline & & & & 99mTC-MIBI n=1 & Unknown=1 & & \\
\hline & & 3 & Brain & $\begin{array}{l}\text { MRI } n=2 \\
\text { CT } n=1\end{array}$ & PR & $\begin{array}{l}\text { Alive } n=2 \\
\text { Unknown } \\
n=1\end{array}$ & $\begin{array}{c}5.5-9 \\
2.5\end{array}$ \\
\hline Tsoli et al. & 2017 & 1 & Brain & $\mathrm{MRI}$ & Resection, RTx & Death & 2 \\
\hline Asare et al. & 2019 & 2 & Liver & Unkown & CTx & $\begin{array}{l}\text { Alive } n=1 \\
\text { Death } \\
n=1\end{array}$ & $\begin{array}{l}\text { Unkown } \\
\qquad n=6\end{array}$ \\
\hline
\end{tabular}

Distant metastases were only considered, if they were localized extracervical, extrathoracic and were not localized in bones. RFA, radiofrequency ablation; EB, embolization; PR, palliative resection; CTx, chemotherapy; RTx, Radiotherapy.

detecting uncommon tumor localization and to avoid repeated cervical surgery.

Furthermore, in our case the selective venous sampling was additionally performed to confirm this uncommon localization. Therefore, selective venous sampling was able to support the localization diagnostics and consecutively, the focused surgical approach we then performed.

\section{MOLECULAR INFORMED SYSTEMIC TREATMENT}

Overall, only few reports have characterized the molecular landscape of PC. Using exome sequencing, Yu et al. (20) in a series of seven cases found an average of 51 somatic variants. This is in stark contrast to the findings in our patient who had high tumor mutational burden (TMBh). TMBh is increasingly recognized as a marker of response to immunotherapy (21) and related to the accumulation of tumoral neoantigens which increase the likelihood of response to inhibition of immune checkpoint molecules.

Molecular guided systemic treatment with pembrolizumab led to stable disease as best response on imaging and a marked clinical and biochemical benefit with a stabilization of serum calcium and drop of PTH for 9 months. Our case demonstrates that in rare tumors, molecular analysis may be useful to detect druggable targets. So far, no case of successful treatment of PC with immune checkpoint inhibitors has been described. 


\section{TARGETED SURGICAL RESECTION PERMITTED LONG-TERM DISEASE CONTROL}

After the first two resections of parathyroid metastases, calcium normalization has been achieved. The patient was subsequently clinically and biochemically disease free for 9 months (at last observation 10/2020). Repeated surgery was able to control serum calcium supporting that every effort should be made to localize the disease and evaluate resectability $(16,17)$. It must be noted that our previous case series suggests that more extended primary surgery (parathyroidectomy and hemithyroidectomy) may be beneficial in lowering the rate of recurrence in general although it is unclear whether this is also true for the very rare distant metastases (7).

\section{PATIENTS PERSPECTIVE}

Our patient has been alive with a "chronic" disease over a long duration which required medical treatment for long periods of time. Uncertainty about prognosis, eventual diagnostic success and risk of surgery have contributed to the disease burden. The present outcome with biochemical remission has significantly improved the well-being of the patient.

\section{CONCLUSION}

Because manifestation of recurrent PC outside of the neck and chest is possible, whole body imaging for tumor localization is useful to allow for focused repeat surgery. The course of the disease in our patient with a recurrence after 12 years after primary surgery suggests that the tumor evaded immune recognition at a certain time point during disease progression which may also explain the unusual systemic spread of the disease. It is tempting to speculate that pembrolizumab treatment inhibited further progression of disease rendering surgical removal effective in a neo-adjuvant manner. A multimodal diagnostic approach and therapy in an interdisciplinary setting is needed for patients with rare endocrine tumors.

\section{REFERENCES}

1. Ryhanen EM, Leijon H, Metso S, Eloranta E, Korsoff P, Ahtiainen P, et al. A nationwide study on parathyroid carcinoma. Acta Oncol (2017) 56(7):9911003. doi: 10.1080/0284186X.2017.1306103

2. Obara T, Fujimoto Y. Diagnosis and treatment of patients with parathyroid carcinoma: an update and review. World J Surg (1991) 15(6):738-44. doi: 10.1007/BF01665308

3. Lee PK, Jarosek SL, Virnig BA, Evasovich M, Tuttle TM. Trends in the incidence and treatment of parathyroid cancer in the United States. Cancer (2007) 109(9):1736-41. doi: 10.1002/cncr.22599

4. Cetani F, Pardi E, Marcocci C. Parathyroid Carcinoma. Front Horm Res (2019) 51:63-76. doi: 10.1159/000491039

5. Harari A, Waring A, Fernandez-Ranvier G, Hwang J, Suh I, Mitmaker E, et al. Parathyroid carcinoma: a 43-year outcome and survival analysis. J Clin Endocrinol Metab (2011) 96(12):3679-86. doi: 10.1210/jc.2011-1571

\section{DATA AVAILABILITY STATEMENT}

The original contributions presented in the study are included in the article/Supplementary Material. Further inquiries can be directed to the corresponding author.

\section{ETHICS STATEMENT}

Written informed consent was obtained from the individual(s) for the publication of any potentially identifiable images or data included in this article.

\section{AUTHOR CONTRIBUTIONS}

CL, NS, and MK designed the study. $\mathrm{AB}$ and RK performed diagnostic imaging and procedures. JR and AW performed surgical procedures. CG supervised all surgical procedures. SK examined all histopathological samples. AS, DH, and SF performed whole exome sequencing. CL, CF, and MK provided data. CL and MK analyzed the data. CL, MF, NS, CG, AS, DH, SF, and MK interpreted the data. CL and MK wrote a manuscript draft. All authors contributed to the article and approved the submitted version.

\section{FUNDING}

This publication was supported by the Open Access Publication Fund of the University of Wuerzburg.

\section{SUPPLEMENTARY MATERIAL}

The Supplementary Material for this article can be found online at: https://www.frontiersin.org/articles/10.3389/fendo.2021. 643328/full\#supplementary-material

6. Witteveen JE, Haak HR, Kievit J, Morreau H, Romijn JA, Hamdy MA, et al. Challenges and pitfalls in the management of parathyroid carcinoma: 17-year follow-up of a case and review of the literature. Horm Cancer (2010) 1(4):20514. doi: 10.1007/s12672-010-0042-6

7. Lenschow C, Schragle S, Kircher S, Lorenz K, Machens A, Dralle H, et al. Clinical Presentation, Treatment, and Outcome of Parathyroid Carcinoma: Results of the NEKAR Retrospective International Multicenter Study. Ann Surg (2020). doi: 10.2139/ssrn.3541131

8. Cetani F, Pardi E, Marcocci C. Update on parathyroid carcinoma. J Endocrinol Invest (2016) 39(6):595-606. doi: 10.1007/s40618-016-0447-3

9. Campenni A, Ruggeri RM, Sindoni A, Giovinazzo S, Calbo E, Ieni A, et al. Parathyroid carcinoma presenting as normocalcemic hyperparathyroidism. J Bone Miner Metab (2012) 30(3):367-72. doi: 10.1007/s00774-011-0344-y

10. Wang P, Xue S, Wang S, Lv Z, Meng X, Wang G, et al. Clinical characteristics and treatment outcomes of parathyroid carcinoma: A retrospective review of 234 cases. Oncol Lett (2017) 14(6):7276-82. doi: 10.3892/ol.2017.7076 
11. Machado NN, Wilhelm SM. Parathyroid Cancer: A Review. Cancers (Basel) (2019) 11(11):1676. doi: 10.3390/cancers11111676

12. Manente P, Cecchettin M, Infantolino D, Foscolo G, Conte N. Apparently nonfunctioning metastases of parathyroid carcinoma. Tumori (1987) 73 (2):191-3. doi: $10.1177 / 030089168707300218$

13. Qiu ZL, Wu CG, Zhu RS, Xue YL, Luo QY. Unusual case of solitary functioning bone metastasis from a "parathyroid adenoma": imagiologic diagnosis and treatment with percutaneous vertebroplasty-case report and literature review. J Clin Endocrinol Metab (2013) 98(9):3555-61. doi: 10.1210/ jc.2013-2014

14. WEEKLY clinicopathological exercises; parathyroid carcinoma with hyperparathyroidism and with metastases to liver. N Engl J Med (1953) 248 (10):426-32. doi: 10.1056/NEJM195303052481007

15. Lloyd RV, Osamura RY, Klöppel G, Rosai J. WHO classification of tumours of endocrine organs by International Agency for Research. In: RV Lloyd, editor. Cancer, 4th ed, vol. 10 th. World Health Organization (WHO, Genf, Switzerland (2017).

16. Storvall S, Ryhanen E, Bensch FV, Heiskanen I, Kytola S, Ebeling T, et al. Recurrent Metastasized Parathyroid Carcinoma-Long-Term Remission After Combined Treatments With Surgery, Radiotherapy, Cinacalcet, Zoledronic Acid, and Temozolomide. JBMR Plus (2019) 3(4):e10114. doi: 10.1002/ jbm4.10114

17. Evangelista L, Sorgato N, Torresan F, Boschin IM, Pennelli G, Saladini G, et al. FDG-PET/CT and parathyroid carcinoma: Review of literature and illustrative case series. World J Clin Oncol (2011) 2(10):348-54. doi: 10.5306/wjco.v2.i10.348

18. Deandreis D, Terroir M, Al Ghuzlan A, Berdelou A, Lacroix L, Bidault F, et al. (1)(8)Fluorocholine PET/CT in parathyroid carcinoma: a new tool for disease staging? Eur J Nucl Med Mol Imaging (2015) 42(12):1941-2. doi: 10.1007/ s00259-015-3130-6

19. Hatzl M, Roper-Kelmayr JC, Fellner FA, Gabriel M. 18F-Fluorocholine, 18F-FDG, and 18F-Fluoroethyl Tyrosine PET/CT in Parathyroid Cancer. Clin Nucl Med (2017) 42(6):448-50. doi: 10.1097/RLU.0000000000001652

20. Yu W, McPherson JR, Stevenson M, van Eijk R, Heng HL, Newey P, et al. Whole-exome sequencing studies of parathyroid carcinomas reveal novel PRUNE2 mutations, distinctive mutational spectra related to APOBECcatalyzed DNA mutagenesis and mutational enrichment in kinases associated with cell migration and invasion. J Clin Endocrinol Metab (2015) 100(2):E360-4. doi: 10.1210/jc.2014-3238

21. Passaro A, Stenzinger A, Peters S. Tumor Mutational Burden as a Pan-cancer Biomarker for Immunotherapy: The Limits and Potential for Convergence. Cancer Cell (2020) 38(5):624-5. doi: 10.1016/j.ccell.2020.10.019

Conflict of Interest: The authors declare that the research was conducted in the absence of any commercial or financial relationships that could be construed as a potential conflict of interest.

Copyright $\odot 2021$ Lenschow, Fuss, Kircher, Buck, Kickuth, Reibetanz, Wiegering, Stenzinger, Hübschmann, Germer, Fassnacht, Fröhling, Schlegel and Kroiss. This is an open-access article distributed under the terms of the Creative Commons Attribution License (CC BY). The use, distribution or reproduction in other forums is permitted, provided the original author(s) and the copyright owner(s) are credited and that the original publication in this journal is cited, in accordance with accepted academic practice. No use, distribution or reproduction is permitted which does not comply with these terms. 